\title{
Entwicklung der ärztlichen Traditionellen Chinesischen Medizin (TCM)/Akupunktur in der Schweiz
}

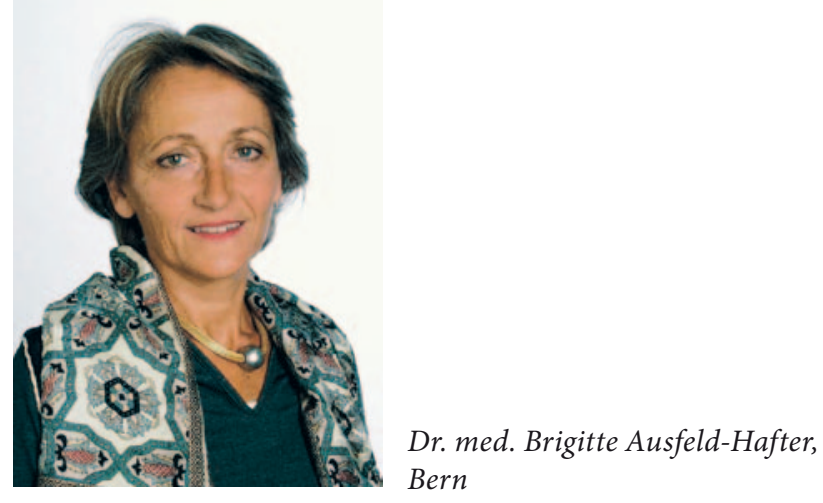

Dieser Artikel gibt eine Übersicht zur Entwicklung der TCM/Akupunktur in der Schweiz seit den 60er-Jahren. Als Dozentin für TCM/Akupunktur an der Kollegialen Instanz für Komplementärmedizin KIKOM gebe ich vor allem einen Einblick in die Tätigkeiten dieser im akademischen Umfeld einzigartigen Instanz.

In den 60er-Jahren kam die Akupunktur als Therapieangebot über den Umweg aus dem von der «Kulturrevolution» gebeutelten China nach Vietnam und später nach Frankreich und in die Schweiz. In den 80er-Jahren wurde von Deutschland her kommend die chinesische Arzneitherapie in der Schweiz heimisch und die von Dr. Paul Nogier entwickelte Aurikulomedizin konnte in Schweizer Praxen aufgenommen werden. Anfang der 90er-Jahre inaugurierte die Universität Zürich einen Lehrstuhl für Komplementärmedizin. Der Werdegang der TCM in der Schweiz ist im Buch «China in der Schweiz» [1] anschaulich dargestellt.

Am 1. Juni 1995 war es soweit: Die Kollegiale Instanz für Komplementärmedizin KIKOM bekam an der Universität Bern ein Lehrstuhläquivalent (www.kikom.unibe.ch). Es wurden Dozenten für die vier komplementärmedizinischen Richtungen TCM/Akupunktur, Anthroposophische Medizin, Klassische Homöopathie und Neuraltherapie zu je $25 \%$ angestellt. Seither ist die TCM in der Schweiz auch im akademischen Bereich vertreten.

Jedem der Viertels-Professoren steht eine 50\%-Stelle für Assistenten sowie eine 50\%-Stelle für das Sekretariat zu. Die Räumlichkeiten der KIKOM befinden sich im Imhoof-
Pavillon auf dem Areal des Inselspitals. Die KIKOM ist mit den Aufgaben der Lehre, der Forschung sowie Dienstleistungen beauftragt. Die TCM/Akupunktur, ebenso wie alle anderen komplementärmedizinischen Richtungen der KIKOM, ist im Bereich der Lehre in das für Studierende fakultative Curriculum des 1., 2., 3. und 5. Studienjahres aufgenommen worden und kann für einen Monat im Wahlstudienjahr belegt werden. Im Frühlingssemester 2010 wurde zum ersten Mal eine für alle Medizinstudierenden im 3. Jahr obligatorische Konzeptvorlesung von drei Stunden angeboten, die mit zwei Fragen in der anschliessenden Multiple-Choice-Prüfung vertreten ist. Darüber hinaus stehen Fortbildungen, Seminare und Gastvorlesungen auf dem Programm. Seit 1998 erscheint jährlich die Ringvorlesung «Komplementäre Medizin im interdisziplinären Diskurs» in Buchform beim Peter Lang Verlag (www.peterlang.com).

Die Anbindung an die Universität hat zur Folge, dass wissenschaftliche Studien durchgeführt werden können. Dies allerdings mit erheblichen Schwierigkeiten, da der Mittelbau, es handelt sich um Oberärzte, in der KIKOM nicht vertreten ist. Seit einigen Jahren werden daher die wissenschaftlichen Mitarbeiter durch Drittmittel finanziert, die dann ihr Wissen und Können je nach Erfolg der Drittmittelbeschaffung während der Anstellungsdauer einbringen. Lange andauernde Stellenbesetzungen sind in der Forschung praktisch unabdingbar.

Ein anderes Problem besteht bei der Besetzung der Assistentenstelle für die Patientenbetreuung: Ein zu 50\% angestellter Arzt soll von einem zu 25\% angestellten Dozenten angeleitet werden - da sind innovative Lösungen gefordert.

In meinem Departement wurde in den letzten 15 Jahren im Bereich der Forschung viel gearbeitet. Da ist zunächst die Zusammenarbeit mit dem Projekt Evaluation Komplementärmedizin PEK [2] zu erwähnen, aus welcher in der Folge diverse Artikel in Peer-reviewed-Zeitschriften resultierten [3]. Vor einigen Jahren wurde im Fachbereich TCM/ Akupunktur eine grössere randomisierte, doppelblinde Studie zur Wirkung der Lasernadeltherapie bei Dysmenorrhoe durchgeführt [4]. Im Februar 2010 stand der Start einer ebenfalls grossen klinischen Studie zum Thema

Agenda UNION

Vorstandssitzung UNION Vorstandssitzung UNION Vorstandssitzung UNION

Delegiertenversammlung UNION 2011
Donnerstag, 16. September 2010 (Nachmittag); Ort wird noch bekannt gegeben Donnerstag, 21. Oktober 2010 (Nachmittag); Ort wird noch bekannt gegeben Donnerstag, 18. November 2010 (Nachmittag); Ort wird noch bekannt gegeben Donnerstag, 5. Mai 2011 (Nachmittag); Ort wird noch bekannt gegeben

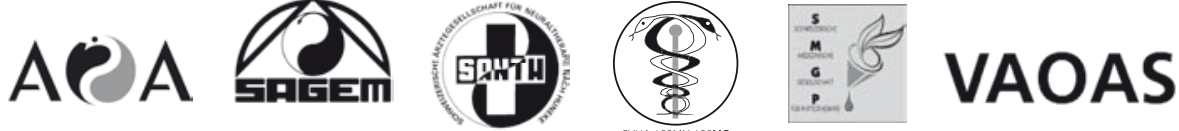


Menopausebeschwerden nach unzähligen misslichen Rückschlägen in der Prüfphase an [5].

Es sind auch diverse Fragebogenstudien durchgeführt worden, z.B. der vor der Abstimmung vom 17. Mai 2009 erschienene Artikel «Komplementärmedizin in Schweizer Praxen der Grundversorgung» [6]. Diese Umfrage bei 750 Ärzten in der Schweiz wurde von der UNION schweizerischer komplementärmedizinischer Ärzteorganisationen gesponsert. Insgesamt wurden bisher sieben Dissertationen unter meiner Leitung abgeschlossen, vier weitere sind in Bearbeitung und zwei Masterarbeiten werden voraussichtlich 2010 fertig gestellt. Das Fazit der langjährigen Forschungstätigkeit lautet: Vier klinische Studien durchgeführt, sechs Fragebogenstudien abgeschlossen, sieben weitere in Bearbeitung. Die Publikationen dieser diversen Arbeiten sind alle in Peer-reviewed-Zeitschriften erschienen.

Viele Forschungsarbeiten entstanden in Zusammenarbeit mit anderen Instituten des Inselspitals; die dafür notwendige Finanzierung wurde vornehmlich durch die Akquirierung von Drittmitteln sichergestellt. Leider ist es bisher nicht möglich gewesen, mit mehr als einem ChinaMed Zentrum (das erste wurde 1998 in Baden AG eröffnet) ein Forschungsprojekt zu erarbeiten. Die KIKOM hat in den letzten Jahren eine Vernetzung auf internationaler Ebene erreichen können; ungezählt sind die Kongressbesuche mit Referaten und Posterbeteiligungen. Dies ist umso erfreulicher, da die Schweiz nur durch eine erfolgreiche Vernetzung der Forschung ihre Stimme in Europa einbringen kann.

Nebst mehr als 50 Buch- und Zeitschriftenbeiträgen war es mir immer ein Anliegen, die TCM auch einem grösseren Publikum zugänglich zu machen. Aus diesem Grund hat jeweils einer der vier Dozenten seit 1997 im Herbstsemester eine für alle offene Vorlesungsreihe «Komplementäre Medizin im interdisziplinären Diskurs» durchgeführt. Die vierte von mir organisierte Reihe hat im Herbstsemester 2009 zum Thema «Chronobiologie» stattgefunden.

Der von mir initiierte Förderpreis für Komplementärmedizin «[5]×[1]» unterstützt die Forschung und Lehre in der Schweiz und wird seit 2008 alljährlich während des ASA TCM-Kongresses in Basel würdevoll verliehen [7].

Die Zusammenarbeit mit der Assoziation Schweizerischer Ärztegesellschaften für Akupunktur und Chinesische Medizin (ASA) [8], die am 6. Juni 1998 gegründet wurde, hat sich in all den Jahren als effizient erwiesen. Die ASA hat als erste komplementärmedizinische Gesellschaft ein Weiter- und Fortbildungscurriculum erarbeitet, das zur Erlangung des Fähigkeitsausweises dient. Es wurde 1998 von der Kommission für Weiter- und Fortbildung der FMH genehmigt. Das Curriculum beinhaltet drei Ausbildungsmodule à 80 Stunden sowie 120 Stunden Weiter- bildung zur Vertiefung der Grundlagen und ist an eine regelmässige Fortbildung von mindestens 18 Stunden innerhalb von zwei Jahren gebunden.

Seit 2005 gibt es unter dem Patronat der KIKOM und in Zusammenarbeit mit den Fachgesellschaften die Ausbildungsmodule Akupunktur I und II, das Modul Aurikulomedizin und nun auch das Modul chinesische Arzneilehre als Angebot für Studierende und Ärzte. Die Kurse werden im Inselspital durchgeführt.

\section{Ausblick}

Meine Pensionierung steht im Jahr 2012 an; mein Nachfolger wird durch eine fakultäre Kommission der Universität Bern gesucht. Das Curriculum eines potenziellen Nachfolgers sollte einige Publikationen enthalten, denn die Universität legt Wert auf wissenschaftliche Tätigkeit. Meine Aufgaben als Dozentin sind mannigfaltig und brauchen viel Herzblut, Initiative und Durchstehungsvermögen, und dies alles bei einem grossen Arbeitspensum. Die wissenschaftliche Tätigkeit, die es mir ermöglicht, Fragestellungen zu ersinnen, deren Beantwortung in der Praxis etwas bewirken kann, hat mich in den letzten Jahren immer erfreut und gefordert. Die Zusammenarbeit mit meinen Kollegen der KIKOM war und ist mir sehr wichtig. Gern stehe ich möglichen Interessenten für Auskünfte über meine Nachfolge zur Verfügung.

\section{Kontakt}

Dr. med. Brigitte Ausfeld-Hafter Dozentin für TCM/Akupunktur Kollegiale Instanz für Komplementärmedizin KIKOM Imhoof-Pavillon, Inselspital, 3010 Bern, Schweiz brigitte.ausfeld-hafter@kikom.unibe.ch

\section{Literatur}

1 Iten F, Saller R: Traditionelle Chinesische Medizin in der Schweiz; in Hugger P (Hrsg): China in der Schweiz. Zwei Kulturen in Kontakt. Zürich, Offizin, 2005, pp 177-200.

2 Melchart D, Mitscherlich F, Amiet M, Eichenberger R, Koch P: Programm Evaluation Komplementärmedizin - Schlussbericht Bern. Bundesamt für Gesundheit, 2005; www.bag.admin.ch/themen/krankenversicherung/ 00263/00264/04102/index.html?lang = de.

3 Michlig M, Ausfeld-Hafter B, Busato A: Patient satisfaction with primary care: a comparison between conventional care and traditional Chinese medicine. Complement Ther Med 2008;16:350-358.

4 Kempf D, Berger D, Ausfeld-Hafter B: Lasernadel-Akupunktur bei Frauen mit Dysmenorrhoe: Eine randomisierte kontrollierte doppelblinde Pilotstudie. Forsch Komplementmed 2009;16:6-12.

5 Ausschreibung für Probandinnen auf der Homepage der KIKOM; www.kikom.unibe.ch.

6 Déglon-Fischer A, Barth J, Ausfeld-Hafter B: Komplementärmedizin in Schweizer Praxen der Grundversorgung. Forsch Komplementmed 2009; 16:251-255; www.kikom.unibe.ch/unibe/content/index_ger.html.

7 Ausschreibung des [5]×[1] Preises auf der Homepage der KIKOM.

8 Sekretariat ASA, Postfach, 8575 Bürglen, Schweiz. www.akupunktur-tcm.ch. 